\title{
Adaptive Subdivision for Hierarchical Non-rigid Registration of Multi-modal Images Using Mutual Information
}

\author{
Adrian Andronache, Philippe Cattin, and Gábor Székely \\ ETH Zurich - Computer Vision Laboratory, \\ Sternwartstrasse 7, CH - 8092, Zurich \\ \{andronache, cattin, szekely\}@vision.ee.ethz.ch
}

\begin{abstract}
In this paper we present an enhanced method for non-rigid registration of volumetric multi-modal images using Mutual Information (MI). Based on a hierarchical subdivision scheme, the non-rigid matching problem is decomposed into numerous rigid registrations of sub-images of decreasing size. A thorough investigation revealed limitations of this approach, caused by a peculiar behavior of MI when applied to regions covering only a limited number of image pixels. We examine and explain the loss of MI's statistical consistency along the hierarchical subdivision. We also propose to use information theoretical measures to identify the problematic regions in order to overcome the MI drawbacks. This does not only improve the accuracy and robustness of the registration, but also can be used as a very efficient stopping criterion for the further subdivision of nodes in the hierarchy, which drastically reduces the computational costs of the entire registration procedure.
\end{abstract}

\section{Introduction}

Medical imaging has been developing extremely fast during the last decades, bringing new technologies to improve pre-operative planning and intra-operative navigation. All the related procedures are using a large number of digital images taken at different time intervals or by different radiological modalities. Medical images are usually containing complementary information and their proper integration is often required. The crucial step in this fusion process is the registration procedure to ensure that the images of interest are in a sufficiently good spatial alignment. Several surveys and textbooks (e.g. [1 and references therein) have already been published providing a broad and general overview and analysis of the related problems and techniques proposed in the literature.

Among other, two key elements are to be mentioned in the context of image registration: the measure used to quantify the similarity between the images to be matched, and the spatial transformation that aligns the images. The introduction of MI as a similarity measure [2,3] has especially influenced the development of intensity-based image registration due to its inherent ability to deal with multi-modal matching problems 4]. Because of unavoidable deformations 
of the anatomy between consecutive scanning procedures, classical rigid, affine or projective registration techniques can only be used in limited special cases. Non-rigid registration procedures, capable to deal with more localized spatial changes, are therefore in the focus of current research and development. Likar and Pernuš proposed in [5] a hierarchical subdivision strategy that decomposes the non-rigid matching problem into an elastic interpolation of numerous local rigid registrations of sub-images of decreasing size.

Starting from the original 2D version of the hierarchical strategy, we improved the algorithm presented in [5] and extended it for volumetric data. In this paper, we first present an analysis of difficulties emerging when using this hierarchical registration scheme. Subsequently, solutions to overcome these drawbacks are presented based on a new method to detect noisy patches or regions of homogeneous intensity within an image. Finally, the performance of our method is compared with the original algorithm [5].

\section{Method}

Even though MI was proven to be a very robust and reliable similarity measure for intensity-based registration of multi-modal images, numerous problems have to be faced if it is applied to small-size images, compromising its usefulness for subdivision schemes. These problems have been identified in connection with either interpolation artifacts or inherent limitations of the MI definition. These difficulties are strongly coupled with the calculation of parameters for the discrete intensity probability distribution estimated by histograms. Therefore, they are increasingly disturbing when the size of the sub-images become smaller with the successive hierarchical subdivision. This effect is, however, less pronounced for $3 \mathrm{D}$ data.

The interpolation artifacts have been broadly analyzed in the literature and different solutions have been proposed [6]7. In order to minimize their influence, Likar and Pernuš artificially increased the number of image samples used for histogram generation by incorporating the global joint distribution as a prior. As a consequence, the statistical reliability of MI is increased. For small patch sizes, however, the use of prior information estimated from the entire image may lead to false maxima in the MI goal function. Therefore, we propose to estimate the prior only from a surrounding area relative to the size of the sub-image.

As another consequence of the successive image splitting, patches of low structural content may appear that often lead to morphologically inconsistent local registrations with a low MI response.

Likar and Pernuš suggested to identify such patches by thresholding the MI value and to exclude them from the local adjustment process. However, this way structured sub-images with low MI value, which still can be registered in a morphologically consistent way, will be prevented to become properly adjusted. At the same time, structureless patches may be retained as according to our observations the MI significantly increases when these start to overlap a structure in the reference image. 
It is well known in information theory [8], that if two signals are statistically independent then their MI is reaching its minimum possible value, namely zero. Therefore, one would expect that by shifting a structureless noisy patch around its initial position, the similarity measure has a small response. Surprisingly, preliminary experiments clearly demonstrated that even though MI is small, it starts to increase as soon as the structureless patch overlaps a region of higher structural content, leading to wrong local registrations, that a thresholding technique or a regularization procedure may fail to detect or correct. The problem is even more pronounced in the context of multi-modal image registration when not all tissue details can be seen in all modalities, like in the case of CT-MR cross-registration.

The following experiment shows this behavior on a simple one-dimensional case. Let us consider two signals $A$ and $B$ depicted in Fig.1(a). We generated the reference signal $A$ by adding white noise to a step function. The floating signal $B$ consists of white noise, and is statistically independent of $A$. Using the basic definition, we can calculate the MI between the two signals as a function of the displacement when the floating signal is translated along the reference signal. The none-zero baseline of the MI, clearly identifiable in Fig. 1(a), can be explained by a combination of two different effects. One is rooted in the difficulty to observe strict independence between signals represented by a finite number of discrete samples. On the other hand, it is well known in the information theory [9] that at the transition from continuous differential entropy to discrete entropy there is systematic bias by an error term depending on the size of the quantization bins used for histogram generation. This theorem does only apply to the marginal entropies $H(A)$ and $H(B)$. We are not aware of any results on deriving a similar relation for the joint entropy $H(A, B)$. Clearly for strictly independent signals $\mathrm{A}$ and $\mathrm{B}$ the quantization error of the discrete entropy would cancel out. This is, however, not the case if the independency condition is perturbed.

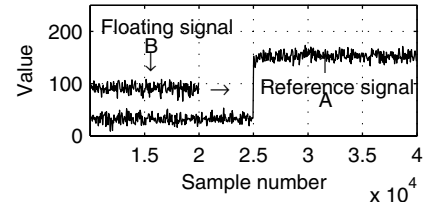

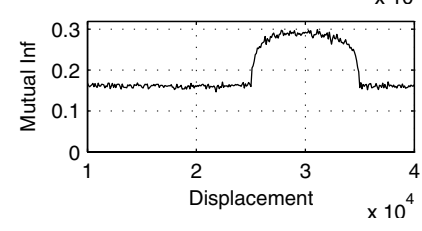

(a)

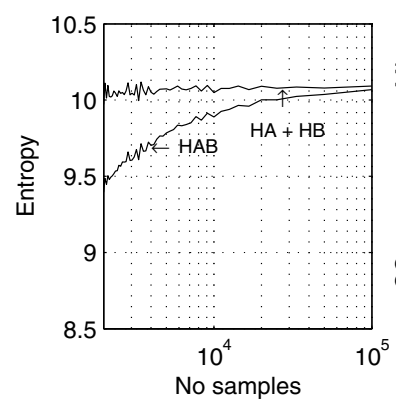

(b)

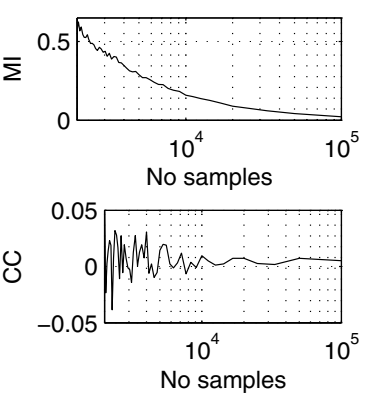

(c)

Fig. 1. (a) Experiment, demonstrating the behavior of MI in the presence of noise, showing the original test signals (top) and the response of MI (bottom) when the floating signal $B$ is shifted over the reference signal $A$. (b) The dependency of the entropies $H(A)+H(B)$ and $H(A, B)$ on the number of samples in the signals. (c) The corresponding dependency of MI and cross-correlation coefficients on the number of samples. 
To further investigate the problem, numerical experiments have been carried out showing the dependency of the entropies on the sample size. The results are shown on Fig. 1(b,c). The cross correlation (CC) graph clearly shows, as expected, that the statistical independence of the two finite, discrete random signals improves with the sample length. Figure1(b), on the other hand, shows a very interesting property of the entropies. While the marginal entropies $H(A)$ and $H(B)$ are only slightly influenced by the sample length and very quickly reach the theoretically predicted value for discrete entropy, the joint entropy $H(A, B)$ requires substantially more samples to show a similarly stable behavior.

Relating this observation to the test signals from Fig. 1(a) it is obvious, that once the floating signal B starts to overlap the step, we get a bi-modal distribution for both $H(A)$ and $H(A, B)$, while $H(B)$ remains constant. The number of available samples needs then to be distributed among two separated distributions for the marginal entropy $H(A)$ and the joint entropy $H(A, B)$. As can be clearly seen from the above graph, the joint entropy $H(A, B)$ decreases much faster than the marginal entropy $H(A)$, necessarily leading to the observed strong increase of the MI. The same behavior has been also noticed when using the normalized mutual information as defined in [10].

As discussed previously, a simple thresholding of the MI during the matching process does not offer a satisfactory solution to the identified problems. Therefore, a new method is necessary, purely relying on the structural content of the single sub-images, allowing to identify structureless nodes in the subdivision scheme. These have to be excluded from both the registration chain and from the further hierarchical splitting process.

Inspired by the point-pattern analysis, where the main goal is to gain information about the spatial pattern or the variation in the data within a region of interest, we propose to use the spatial autocorrelation coefficient to test the consistency of the sub-images. Among different statistical tests proposed in the literature to determine whether the data are randomly or regularly distributed or clustered, the Moran I coefficient is favored by most of the analysts because it has highly desirable statistical properties [11. For a dataset $X=\left\{x_{i}, i=1 . . N\right\}$ of mean value $E(X)=\bar{x}$, Moran's $I$ is defined as

$$
I=\frac{N}{\sum_{i, j=1}^{N} w_{i j}} \cdot \frac{\sum_{i, j=1}^{N} w_{i j} \cdot\left(x_{i}-\bar{x}\right) \cdot\left(x_{j}-\bar{x}\right)}{\sum_{i=1}^{N}\left(x_{i}-\bar{x}\right)^{2}}
$$

where $W=\left\{w_{i j}\right\}$ is called the contiguity matrix characterizing the amount of interaction between the $i$ and $j$ locations and $N$ is the number of observations.

In order to use Moran's $I$ as an indicator of the structural content in 2D or $3 \mathrm{D}$ sub-images, we chose to use a weighting scheme inversely proportional to the Euclidean distance $d(\cdot, \cdot)$ between the currently inspected pixel at the image coordinates $\boldsymbol{i}=\left\{i_{1}, . . i_{n}\right\} \in \mathbb{N}^{n=2,3}$ and its neighbors. A maximum interaction distance $\mathcal{D} \in \mathbb{N}^{n}$ has to be selected according to the minimal size of the structures to be detected in the image. Changing the linearized index notation in (11) to image coordinates, and denoting the vicinity of size $\mathcal{D}$ around $\boldsymbol{i}$ with 
$\mathcal{V}_{\boldsymbol{i}}^{\mathcal{D}}=\left\{\boldsymbol{j} \in \mathbb{N}^{n}, \forall\left|i_{k}-j_{k}\right| \leq \mathcal{D}_{k}\right.$ and $\left.k=1 . . n\right\}$, then the contiguity matrix can be expressed as

$$
W= \begin{cases}w_{\boldsymbol{i} j}=\frac{1}{d(\boldsymbol{i}, \boldsymbol{j})}, & \forall \boldsymbol{j} \in \mathcal{V}_{\boldsymbol{i}}^{\mathcal{D}} \backslash\{\boldsymbol{i}\} \\ 0, & \text { otherwise }\end{cases}
$$

Denoting with $a_{\boldsymbol{i}}$ the intensity value of the image voxel located at the spatial position $\boldsymbol{i}$ within an image patch $A$ of size $\boldsymbol{N} \in \mathbb{N}^{n}$ and mean value $\bar{a}$, the Moran $I$ becomes

$$
I=\frac{1}{\sum_{\boldsymbol{j} \in \mathcal{V}_{0}^{\mathcal{D}}} w_{\mathbf{0} \boldsymbol{j}}} \cdot \frac{\sum_{\boldsymbol{i} \in \boldsymbol{N}} \sum_{\boldsymbol{j} \in \mathcal{V}_{\boldsymbol{i}}^{\mathcal{D}}} w_{\boldsymbol{i} \boldsymbol{j}} \cdot\left(a_{\boldsymbol{i}}-\bar{a}\right) \cdot\left(a_{\boldsymbol{j}}-\bar{a}\right)}{\sum_{\boldsymbol{i} \in \boldsymbol{N}}\left(a_{\boldsymbol{i}}-\bar{a}\right)^{2}}
$$

Moran's $I$ varies in the interval $[-1,1]$, where the random patterns are characterized by values close to zero. As the associated standard $Z$ value is asymptotically normally distributed [1], a threshold of \pm 1.96 corresponding to the $95 \%$ confidence interval can be chosen to identify random, i.e. structureless patterns. Figure 3(b-c) shows the result of the analysis at the $5^{\text {th }}$ level of the hierarchy of a 2D neuroradiological MR slice.

We incorporated the enhancements discussed above into the $3 \mathrm{D}$ extended version of the hierarchical subdivision scheme described in [5. The Moran information consistency test is applied to every sub-image in order to pass only the relevant image patches to the succeeding rigid registration stage. For all the sub-images failing this consistency test, the hierarchical splitting is stopped. However, for the 3D version, care has to be given to anisotropic voxel dimensions. In order to avoid discontinuities between neighboring leafs the parameter inheritance from one level to the other, used for the initialization, is done by linearly interpolating the transformations of the parent leaf and its surrounding neighbors. The consistency of the identified individual transformations between neighboring sub-images is ensured by a regularization procedure. It imposes spatial constraints on the centroids of the sub-images by defining a distance range between them. The identified outliers are corrected by assigning them the average transformation of all their surrounding sub-images. Thin-plate splines (TPS) [12] are used to calculate the final deformation field by densely interpolating the regularized local transformations of the sub-images over the whole image domain.

\section{Results}

For all the experiments, the registration algorithm was using the following parameter settings: (a) the two-dimensional histogram was generated using 256 bins; (b) the prior joint probability was estimated locally only from the direct neighboring sub-windows; (c) the threshold for the magnitude of the standard $Z$ value of Moran's $I$ coefficient used in the information consistency test was set to 1.96 ; (d) the contiguity matrix was calculated according to a maximum distance of 3 pixels $((7 \times 7)$ or $(7 \times 7 \times 7)$ for the two- and three-dimensional case respectively), considering also the voxel anisotropy. 
We illustrate the performance of our method on a CT-MR cross-registration example. The reference image is a $512 \times 512 \times 46 \mathrm{CT}$ scan of the head with $0.39 \times 0.39 \times 0.6 \mathrm{~mm}^{3}$ voxel dimension and the floating image is a $512 \times 512 \times 28$ MR scan with voxels of the size $0.5 \times 0.5 \times 1.0 \mathrm{~mm}^{3}$. Figure 2 visualizes details of our non-rigid registration method in a region where elastic deformation is needed to correct for both MR susceptibility artifacts (e.g. within the left sphenoid sinus) and tissue deformation (e.g. the left ear) between the two acquisitions. In order to better compare the results between a global rigid registration (Fig. 2 (c,d)) and after applying the enhanced hierarchical registration process (Fig. $2(\mathrm{e}, \mathrm{f})$ ), the outline of the head and of the left sphenoid sinus is extracted from the floating $\mathrm{MR}$ volume and overlaid on the reference CT image. The remaining deviations of the two contours are caused by both the spatial constraints imposed by the regularization of the deformation field and the size of the smallest sub-image $(16 \times 16 \times 8)$ given by the number of hierarchical splitting levels.

Figure 3 shows the performance of our enhanced algorithm in comparison with the original method [5] for a two-dimensional registration example. The reference image (Fig. 3(a)) shows one slice from the aforementioned CT scan of the head and the floating image shows the corresponding transversal slice of the rigidly registered MR volume. A comparison between Fig. 33(f) and Fig. 3. (g) clearly shows the favorable effect of using Moran's test when the local registration is dealing with structureless sub-images. Depending on the number of structureless sub-images found, the new algorithm can perform significantly faster than the original method (e.g. approximately a factor of two for this experiment). Even more, the Moran's consistency test incorporated into the enhanced algorithm allows us to go further with the hierarchical subdivision down to sub-images of $16 \times 16$ pixels, while Likar and Pernuš reported a minimum sub-image size of $95 \times 64$ pixels.

To quantify the improvement of the registration, we used an empirical procedure proposed by Ion Pappas et al. 13] to measure the performance of

(a)

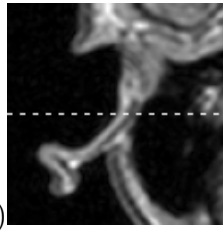

(b)

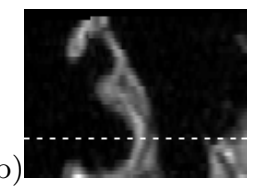

(c)

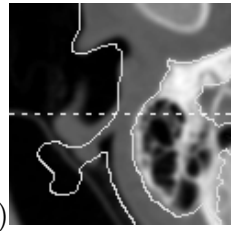

(d)

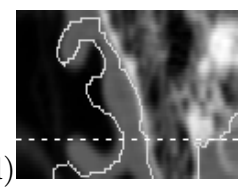

(e)

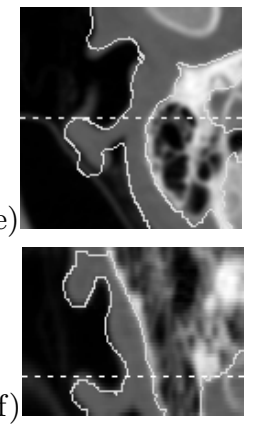

Fig. 2. Result of a CT-MR cross-registration. (a) Transversal and (b) coronal sections of the region of interest in the initial floating MR volume. (c-f) Corresponding sections in the reference CT volume, overlaid with the contours of the head and of the sphenoid sinus after a global rigid (c,d) and after the full hierarchical (e,f) registration. Note: The dashed lines mark the position of the cutting planes. 
(a)

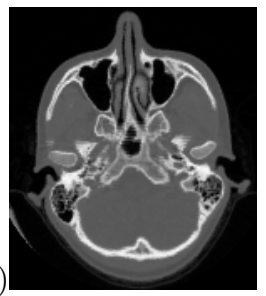

(b)

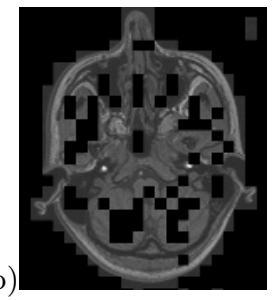

(c)

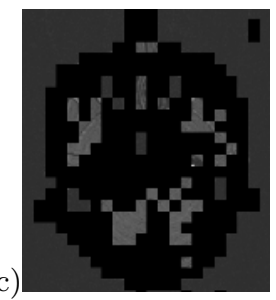

(d)

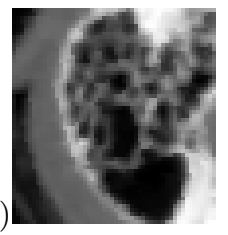

(e)

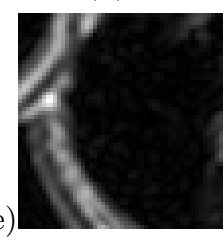

(f)
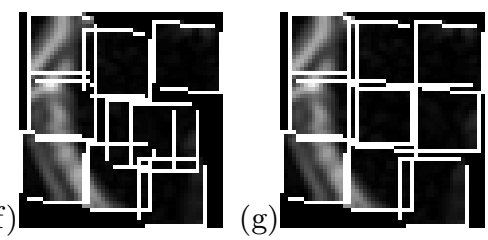

Fig. 3. Registration details of the sphenoid sinus in the left temporal bone at the $5^{\text {th }}$ level of the hierarchy. (a) The reference CT. (b) and (c) The result (cropped images) of Moran's consistency test on the floating MR, divided in $32 \times 32$ sub-images of $16 \times 16$ pixels. The examined region, $((\mathrm{d})$ on the $\mathrm{CT}$ and $(\mathrm{e})$ on the MR) consists of $3 \times 3$ sub-images. (f) Depicts the final position of each image patch after the local rigid registration, while $(\mathrm{g})$ shows the result after applying the local rigid registration only to those MR patches which passed Moran's test. The consistency check clearly prevented the two middle patches from being pulled towards structures in the reference CT.

multimodal registration procedures. Similar to [14, the method estimates the percentage of overlapping volumes segmented from the images of interest. The final score of the registered bony structures using the improved algorithm shows a $2.3 \%$ improvement over the original method of Likar et al.

\section{Conclusions}

In this paper we extended and improved the hierarchical non-rigid registration algorithm relying on the maximization of MI, proposed by Likar and Pernuš. An information consistency test based on Moran's spatial autocorrelation coefficient has been developed and used to detect and eliminate the structureless sub-images from the registration chain, thus avoiding registration errors caused by structurally meaningless extrema of the MI. However, the MI proved to be more robust for the $3 \mathrm{D}$ version of the algorithm due to the larger number of available samples. Moran's test results in a very effective stopping criterion for the hierarchical subdivision process allowing to eliminate errors introduced by problematic structureless sub-images and it also speeds up the entire algorithm.

We see several possible directions for future research. Currently, the hierarchical binary splitting is ignoring the image content. An adaptive positioning of the splitting boundaries, enabling better adaptation to the anatomical structures, would not only further reduce the computational load but also improve image registration quality. This adjustment process could also be supported by using 
Moran's spatial autocorrelation coefficient. Finally, the regularization method could be significantly improved by adapting the corresponding corrections to the certainty of the estimated transformation parameters, which can be measured by their standard deviation estimated using standard error propagation methods.

\section{Acknowledgments}

This work has been supported by the CO-ME/NCCR research network of the Swiss National Science Foundation (http://co-me.ch). We are also grateful to the MEMCenter, University of Bern.

\section{References}

1. Hajnal, J.V., Hill, D.L., Hawkes, D.J.: Medical Image Registration. CRC Press (2001)

2. Maes, F., Collignon, A., Vandermeulen, D., Marchal, G., Suetens, P.: Multimodality image registration by maximization of mutual information. Proc IEEE Workshop Mathematical Methods in Biomedical Image Analysis (1996) 14-22

3. Viola, P., W. M. Wells, I.: Alignment by maximization of mutual information. In: ICCV '95: Proceedings of the Fifth International Conference on Computer Vision. ISBN 0-8186-7042-8, IEEE Computer Society (1995) 16

4. Maes, F., Vandermeulen, D., Suetens, P.: Medical image registration using mutual information. Proc IEEE 91 (2003) 1699-1722

5. Likar, B., Pernus, F.: A hierarchical approach to elastic registration based on mutual information. Image and Vision Computing 19 (2001) 33-44

6. Pluim, J.P.W., Maintz, J.A., Viergever, M.A.: Mutual information matching and interpolation artefacts. Proc SPIE Medical Imaging 3661 (1999) 56-65

7. Ji, J.X., Pan, H., Liang, Z.P.: Further analysis of interpolation effects in mutual information-based image registration. IEEE Transactions on Medical Imaging 22 (2003) 1131-1140

8. Shannon, C.E.: A mathematical theory of communication. Technical report, Bell Laboratories (1948)

9. Cover, T., Thomas, J.: Elements of Information Theory. John Wiley \& Sons (1991)

10. Studholme, C., Hill, D., Hawkes, D.: An overlap invariant entropy measure of 3d medical image alignment. Pattern Recognition 32 (1999) 71-86

11. Cliff, A., Ord, J.: Spatial Autocorrelation. ISBN 0-85086-036-9. Pion Limited: R.J. Chorley and D.W. Harvey, 207 Brondesbury Park, London NW2 5JN (1973)

12. Bookstein, F.L.: Principal warps: Thin-plate splines and the decomposition of deformations. IEEE Trans Pattern Anal Mach Intell 11 (1989) 567-585

13. Pappas, I.P., Malik, P., Styner, M., Liu, J., Caversaccio, M.: New method to assess the registration of ct-mr images of the head. SPIE Medical Imaging 5370 (2004) $14-19$

14. Hellier, P., Barillot, C., Corouge, I., Gibaud, B., Goualher, G.L., Collins, D., Evans, A., Malandain, G., Ayache, N., Christensen, G., Johnson, H.: Retrospective evaluation of intersubject brain registration. Medical Imaging, IEEE Transactions on 22 (2003) 1120- 1130 